
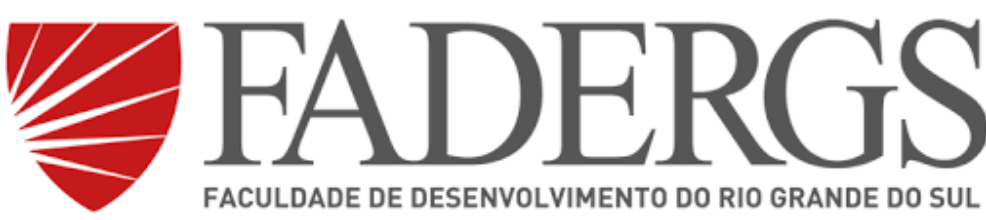

FACULDADE DE DESENVOLVIMENTO DO RIO GRANDE DO SUL

ESCOLA DE SAÚDE E BEM-ESTAR

CURSO DE ENFERMAGEM

AMANDA LUCIANO BARCELLOS

CUIDADOS PALIATIVOS NO CÂNCER DE PULMÃO

PORTO ALEGRE

2018 


\section{CUIDADOS PALIATIVOS NO CÂNCER DE PULMÃO}

Trabalho de conclusão do curso de enfermagem da FADERGS como requisito de aprovação da disciplina de TCC dois. Professor Orientador: Rafael Vercelino

Porto Alegre 


\section{RESUMO}

O cuidado paliativo voltado ao paciente com câncer constitui um alicerce fundamental para o bem-estar da pessoa enferma. O objetivo deste estudo é fazer uma revisão de alguns estudos científicos relacionados com os cuidados paliativos para o paciente com câncer de pulmão. Fez-se, assim, uma coleta de dados nas bases científicas Scielo e Medline, com os critérios de avaliação de artigos escritosem português e inglês, nos anos de 2013 a 2018, e com abordagem somente voltada para adultos. Foram utilizados artigos em inglês e português e excluídos artigos conduzidos com crianças.Dessa forma, foram abordados os seguintes temas: Teoria Humanística,como tendo o principal foco na abordagem de diminuição do sofrimento físico, psicossocial,espiritual e existencial, contudo visando estabelecer uma meta de comunicação abrangente e aberta com o paciente e seus familiares, a fim de promover melhor qualidade de vida ao indivíduo. $\mathrm{Na}$ análise da Teoria do Autocuidado, foi utillizada a Teoria de Dorothea Orem: as práticas de atividades iniciadas e executadas pelo indivíduo em seu própio benefício para manutenção da vida e do seu bemestar. Na Teoria do Cuidado Humano, foi estudada a Teoria de John Watson, a qual ajuda o profissional enfermeiro a fazer a conexão do paciente com o ambiente hospitalar. Também estudamos a Teoria de Martin Heidegger, que enfatiza a cuidado com algo que transcende o simples acompanhamento do paciente, podendo gerar afinidade e respeito, visando,assim, promover uma recuperação da dignidade do indivíduo.

Descritores:Câncer de pulmão. Cuidados paliativos, Qualidade de vida. 


\begin{abstract}
Palliative care for the cancer patient is a fundamental foundation for the well-being of the sick person. The aim of this study is to review some scientific studies related to palliative care for patients with lung cancer. Thus, a data collection was carried out in the scientific bases Scielo and Medline, with the evaluation criteria of articles written in Portuguese and English, from 2013 to 2018, and with an adult-only approach. Use the articles in English and Portuguese, and delete conducted with children.In this way, the following terms were addressed: Humanistic Theory, as having the main focus on the reduction of physical, psychosocial, spiritual and existential suffering, yet aiming to establish a goal of comprehensive and open communication with the patient and his / her relatives, in order to promote a better quality of life for the individual. In the analysis of Self-Care Theory, Dorothea Orem's Theory was used: the practices of activities initiated and performed by the individual in his own benefit to maintain life and well-being. In the Theory of Human Care, John Watson's Theory was studied, which helps the nurse professional to make the patient's connection to the hospital environment. We also study Martin Heidegger's Theory, which emphasizes caring for something that transcends the simple accompaniment of the patient, and can generate affinity and respect, aiming at promoting a recovery of the dignity of the individual.
\end{abstract}

Keyword:Palliative care,Lung cancer, Health care. 


\section{SUMÁRIO}

1 INTRODUÇÃ

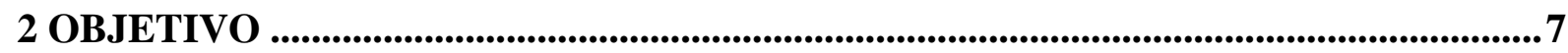

3 METODOLOGIA..........................................................................................................

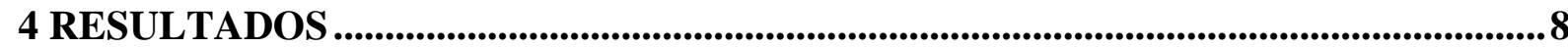

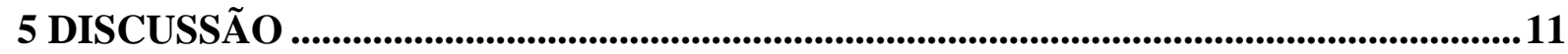

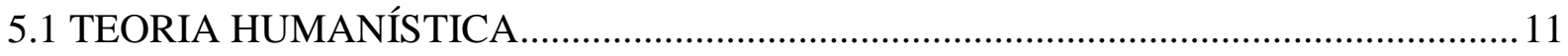

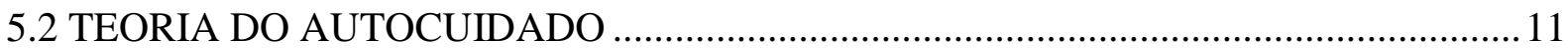

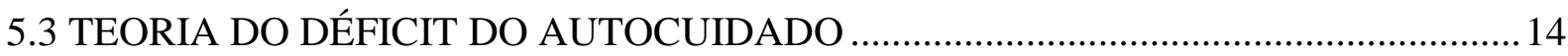

5.4 TEORIA DO CUIDADO HUMANO ………………….......................................... 14

6 CONSIDERAÇÕES FINAIS.................................................................................................. 17

REFERÊNCIAS ............................................................................................18-19 


\section{INTRODUÇÃO}

A neoplasia de pulmão está entre as doenças oncológicas mais comuns no mundo e a que mais mata homens e mulheres dentre os tipos de câncer ${ }^{1}$.Possui um avanço rápido de seus sintomas e exerce um impacto negativo na qualidade de vida do paciente. As características do indivíduo com neoplasia subjacente, que frequentemente apresenta comprometimento da função respiratória, também influenciam a capacidade de fornecer radioterapia com segurança ${ }^{2}$. Nos Estados Unidos,a neoplasia é uma das principais causas de morte por câncer ${ }^{3}$.

Os profissionais da área da enfermagem têm cada vez mais se preocupado com a qualidade na assistência prestada aos seuspacientes, contudo esse fato está relacionado com o exercício legal e com as teorias da enfermagem, sendo assim o profissional enfermeiro deverá basear sua capacidade em cuidados científicos, de maneira que o cuidado possa promover a melhoria na saúde do indivíduo ${ }^{4}$.

Devido à necessidade dessa especialidade, houve resultados na formalização de conceitos e teorias, tendo em vista que essas teorias passaram a ser vistas como um meio de direcionar a enfermagem na busca de seus conhecimentos de atuação em relação a outros profissionais. Assim sendo, os cuidados paliativos têm apresentado uma abordagem que possui como princípio proporcionar suporte aos pacientes e familiares ${ }^{4}$.

Nesse contexto, os cuidados ao ser humano têm a humanização como sendo um item necessário para orientar as ações interdisciplinares, percepções e relações interpessoais. Todavia a enfermagem é a parte principal na atuação do cuidado do indivíduo, onde suas teorias possuem a função de orientar seu exercício, trazendo assim conforto aos seus pacientes ${ }^{4}$.

Os cuidados paliativos consistem em proporcionar uma melhoria na qualidade de vida de uma pessoa doente e a seus familiares, podendo assim aliviar seu sofrimento perante uma doença que pode colocar um fim a sua vida. O diagnóstico tardio do câncer muitas vezes pode dificultar o tratamento com fins curativos, podendo assim diminuir o tempo de sobrevida e também a qualidade de vida do indivíduo. Diante daimpossibilidade de cura da doença, devem ser ajustadas medidas de conforto e que aumentem a qualidade de vida da pessoa através dos cuidados paliativos ${ }^{5}$.

Dentre os cuidados de enfermagem ao paciente oncológico, devem ser promovidos o alivio da dor e também dos sintomas estressantes que possam afetar seu bem-estar e sua qualidade de vida, bem como reafirmar a vida e a morte como sendo um 
processo natural do corpo, proporcionando assim a seus familiares um apoio para que possam lidar com a situação do paciente, pois o mesmo necessita de cuidados especializados como suporte emocional ${ }^{6}$.

No cuidado ao paciente oncológico deve-se priorizar o tratamento holístico, encarando o paciente como um todo, priorizando assim suas necessidades emocionais, espirituais, socioeconômicas, culturais e seus aspectos físicos, além de sua diversidade sobre sua doença. O profissional enfermeiro deverá exercer suas habilidades, requerendo conhecimento científico e habilidades técnicas, para que assim possa exercer uma comunicação adequada entre o paciente e seus familiares ${ }^{6}$.

Promover medidas de cuidado e conforto para o paciente oncológico são aspectos indispensáveis e imprescindíveis para que se possa com eficácia encorajá-lo para que assim fenômenos como a dor sejam diminuídos, fazendo com que o enfermeiro estabeleça um vínculo afetivo entre as partes envolvidas ${ }^{6}$.

Exercer as habilidades interpessoais, bem como a habilidade de comunicação, o ato de ouvir, refletir, e acolher o indivíduo, fazendo com que o mesmo expresse seus sentimentos, também é uma medida de conforto. Dessa forma,é possível proporcionar uma interação entre o paciente e enfermeiro, visando um melhor envolvimento no processo de cuidado, permitindo que eles mesmos possam estabelecer medidas de conforto que venham a suprir suas próprias necessidades ${ }^{6}$.

A dor é um sintoma frequente que está associado ao câncer. A Associação Internacional para o Estudo da Dor aponta a dor como sendo uma sensação de extremo desconforto,uma emoção desagradável que pode estar associada a uma lesão renal ou potencial dos tecidos. A dor é individual e pode ser classificada por cada indivíduo de acordo com suas experiências pessoais ${ }^{7}$.

Para auxiliar no alívio da dor é necessário que o profissional enfermeiro entenda a influência da espiritualidade nesse processo, bem como que a equipe multidisciplinar entenda a importância da espiritualidade no tratamento do câncer ${ }^{8}$.

\section{OBJETIVO}

O presente estudo objetiva realizar uma revisão bibliográfica para avaliar o impacto na qualidade de vida dos cuidados paliativos proporcionados aospacientes oncológicos, especificamente com relação ao câncer de pulmão. 


\section{METODOLOGIA}

O presente estudo consiste em uma inspeção de literatura de natureza bibliográfica. Foram utilizadas as fontes de dados Scielo e Medline para esta revisão. Avaliados artigos cientifícos dos anos de 2013 a 2018,utilizando assim os seguintes descritores: Qualidade de vida, câncer de pulmão e cuidados paliativos.Fizeram parte desse estudo artigos em português e inglês, excluídos artigos com linguagem repetida ou sem relação adequada com o assunto, restando assim 18 artigos para esta revisão.

\section{RESULTADOS}

$\mathrm{Na}$ primeira busca, foram encontrados por fonte eletrônica546 artigos; na segunda busca foram encontrados 300 artigos, sendo 100 artigos com linguagem repetida e sem relação específica com o assunto; por fim,100 artigos de teses ou sem relação com o assunto e outros. Utilizados artigos em português e em inglês, restando assim somente 18 artigos para realização desta revisão.

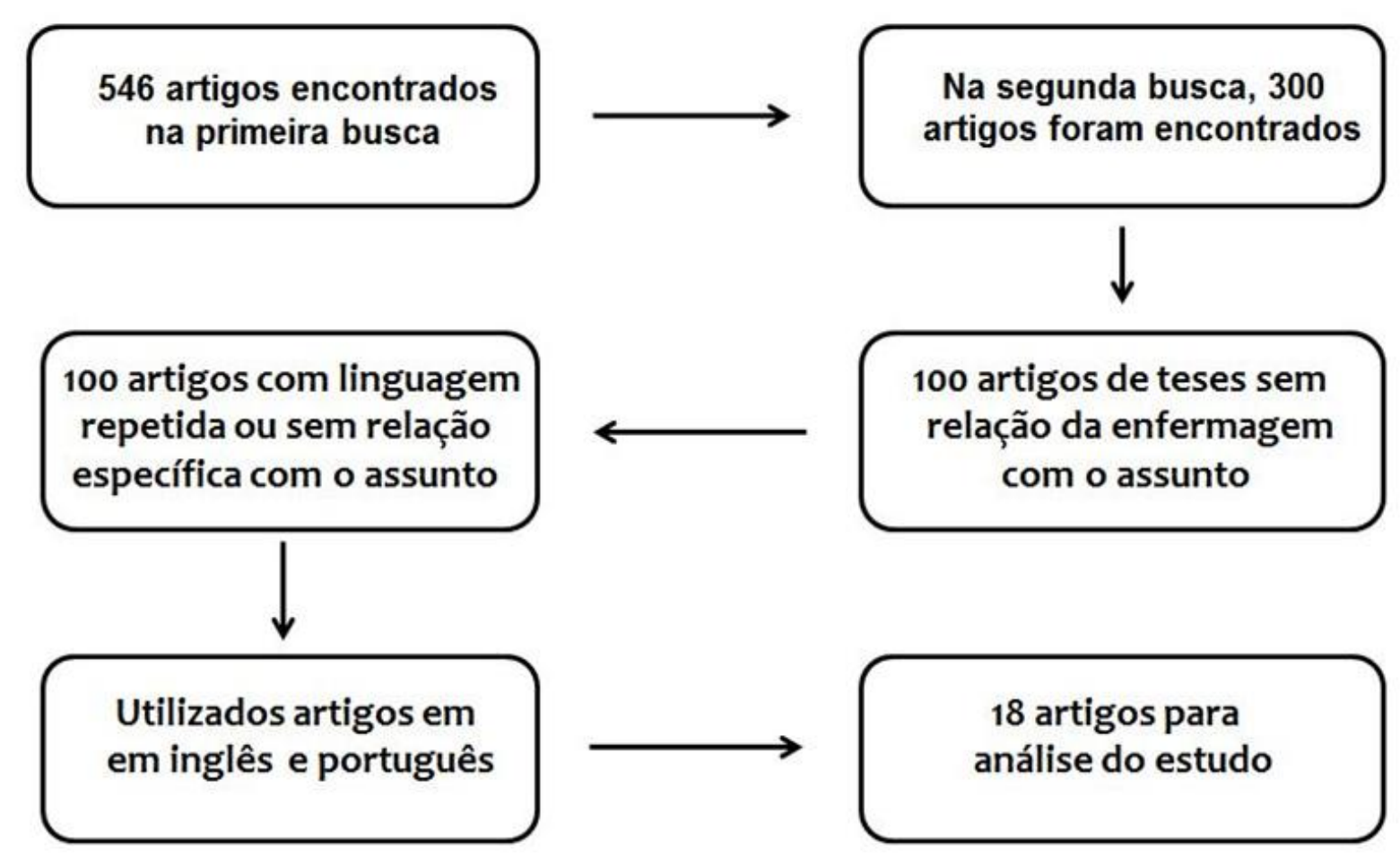

Figura 1 - Organograma da busca nas bases de dados. 
No quadro abaixo, serão mostrados os respectivos autores, títulos e suas teorias de atuação sobre o tema de cuidados paliativos para o paciente com câncer de pulmão.

Quadro 1-Trabalhos selecionados para revisão.

\begin{tabular}{|c|c|c|}
\hline AUTOR & TÍTULO & OBJETIVO DA PESQUISA \\
\hline 1- MASEL EVA (2017) & 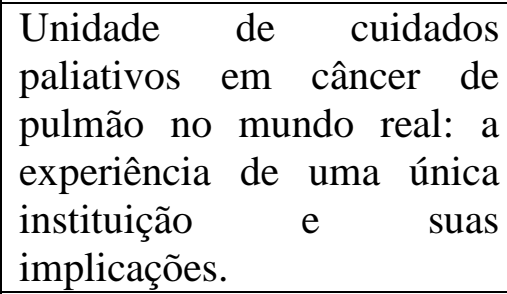 & $\begin{array}{l}\text { Por meio dos cuidados paliativos, } \\
\text { oferecer melhora na qualidade de } \\
\text { vida do paciente com câncer de } \\
\text { pulmão. }\end{array}$ \\
\hline 2 - VINOD (2014) & $\begin{array}{l}\text { Padrão internacional de } \\
\text { prática e radioterapia para } \\
\text { câncer de pulmão. }\end{array}$ & $\begin{array}{l}\text { Por meio da radioterapia, } \\
\text { proporcionar uma melhora na } \\
\text { qualidade de vida do indivíduo } \\
\text { com câncer de pulmão. }\end{array}$ \\
\hline $\begin{array}{l}3 \\
\text { MITCHELL,KENNEDY } \\
(20014)\end{array}$ & $\begin{array}{l}\text { Obstrução maligna das vias } \\
\text { aéreas. }\end{array}$ & $\begin{array}{l}\text { Através dos cuidados paliativos } \\
\text { oferecer uma melhora na } \\
\text { qualidade de vida do paciente. }\end{array}$ \\
\hline $\begin{array}{l}4-\text { SILVA ET AL. } \\
(2018)\end{array}$ & 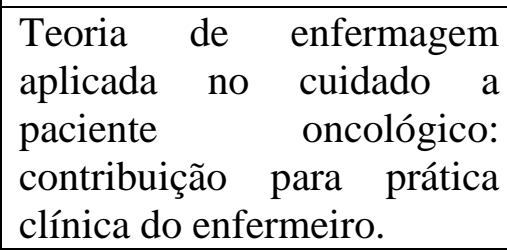 & $\begin{array}{l}\text { Proporcionar melhora na } \\
\text { qualidade de vida do paciente } \\
\text { com câncer por meiodos } \\
\text { cuidados paliativos. }\end{array}$ \\
\hline $\begin{array}{l}5-\text { SILVA ET AL. } \\
(2015)\end{array}$ & $\begin{array}{lcr}\text { Cuidados } & \text { paliativos } & \text { na } \\
\text { assistência } & \text { de } & \text { alta } \\
\text { complexidade } & \text { em } \\
\text { oncologia: percepção } & \text { de } \\
\text { enfermagem. } & & \end{array}$ & $\begin{array}{l}\text { Como melhorar a qualidade do } \\
\text { atendimento do paciente com } \\
\text { câncer através dos cuidados } \\
\text { paliativos. }\end{array}$ \\
\hline $\begin{array}{l}6-\text { SANTOS, G. L, } \\
\text { MATOS (2015) }\end{array}$ & $\begin{array}{l}\text { Cuidados paliativos aos } \\
\text { pacientes oncológicos. }\end{array}$ & $\begin{array}{l}\text { Proporcionar um cuidado } \\
\text { humanizado através dos cuidados } \\
\text { paliativos. }\end{array}$ \\
\hline $\begin{array}{l}7 \text { - ROCHA, A. F. P. ET } \\
\text { AL (2015) }\end{array}$ & $\begin{array}{l}\text { O alívio da dor oncológica: } \\
\text { estratégia contada por } \\
\text { adolescente com câncer. }\end{array}$ & $\begin{array}{l}\text { Alívio da dor física e apoio } \\
\text { emocioanal ao paciente e } \\
\text { familiares. }\end{array}$ \\
\hline $\begin{array}{l}8 \text { - PEREIRA, C. S. et } \\
\text { al. (2016) }\end{array}$ & $\begin{array}{l}\text { Significado dos cuidados } \\
\text { paliativos para a qualidade } \\
\text { da sobrevivência do } \\
\text { paciente oncológico. }\end{array}$ & $\begin{array}{l}\text { Propocionar qualidade de vida ao } \\
\text { paciente sobrevivente de câncer. }\end{array}$ \\
\hline 9 - SHINDE (2018) & $\begin{array}{l}\text { Cuidados paliativosde } \\
\text { câncer de pulmão. }\end{array}$ & $\begin{array}{l}\text { Através dos cuidados paliativos } \\
\text { aliviar o sofrimento físico e } \\
\text { psicológico dos pacientes com } \\
\text { câncer. }\end{array}$ \\
\hline
\end{tabular}




\begin{tabular}{|c|c|c|}
\hline $\begin{array}{ll}10- & \text { HERCOS } \\
(2014) & \end{array}$ & $\begin{array}{l}\text { O trabalho dos profissionais em } \\
\text { enfermagem em unidade de } \\
\text { terapia intensiva na assistência ao } \\
\text { paciente oncológico. }\end{array}$ & $\begin{array}{l}\text { Perante os cuidados de } \\
\text { enfermagem, proporcionar um } \\
\text { melhor cuidado aos pacientes } \\
\text { com câncer. }\end{array}$ \\
\hline $\begin{array}{l}11 \text { SCHIMIGUEL } \\
(2015)\end{array}$ & $\begin{array}{l}\text { O acolhimento de } \\
\text { oncológico: uma } \\
\text { bibliográfica. }\end{array}$ & $\begin{array}{l}\text { Através da revisão de literatura, } \\
\text { proporcionar melhor suporte aos } \\
\text { profissionais da área da saúde. }\end{array}$ \\
\hline $\begin{array}{l}12 \\
\text { NASCIMENTO } \\
(2015)\end{array}$ & O familiar do cliente oncológico. & $\begin{array}{l}\text { Dar apoio emocional ao familiar } \\
\text { do paciente oncológico. }\end{array}$ \\
\hline $\begin{array}{l}13-\text { SANTOS E } \\
\text { RAMOS (2017) }\end{array}$ & \begin{tabular}{lccc} 
Da formação & à & \multicolumn{2}{c}{ prática: } \\
importância & das & teorias & do \\
autocuidado & no & processo & de \\
enfermagem & para & melhoria & dos \\
cuidados. & & & \\
\end{tabular} & $\begin{array}{l}\text { Através da teoria do autocuidado, } \\
\text { proporcionar uma melhora na } \\
\text { qualidade de vida do paciente. }\end{array}$ \\
\hline $\begin{array}{l}14-\text { QUEIRÓS } \\
\text { P.J.P. (2014) }\end{array}$ & $\begin{array}{l}\text { Autocuidado:o contributo teórico } \\
\text { de Orem para a disciplina e } \\
\text { profissão de enfermagem }\end{array}$ & $\begin{array}{l}\text { Através da teoria de Orem, } \\
\text { proporcionar cuidado adequado } \\
\text { ao paciente. }\end{array}$ \\
\hline $\begin{array}{l}15-\text { SILVA ET } \\
\text { AL }(2015)\end{array}$ & $\begin{array}{l}\text { Estratégia de autocuidado das } \\
\text { pessoas com doenças oncológicas } \\
\text { submetidas à quimioterapia e a } \\
\text { sua relação com o conforto. }\end{array}$ & $\begin{array}{l}\text { Proporcionar conforto ao paciente } \\
\text { por meio da teoria do } \\
\text { autocuidado. }\end{array}$ \\
\hline $\begin{array}{l}\text { 16-PEDROSA T. } \\
\text { N. ET AL }(2016)\end{array}$ & $\begin{array}{l}\begin{array}{l}\text { Cuidado e } \\
\text { oncologia } \\
\text { profissionais e usuário }\end{array} \\
\text { significados }\end{array}$ & $\begin{array}{l}\text { Mediante a teoria do auto } \\
\text { cuidado, proporcionar cuidado } \\
\text { adequado, evitando assim a } \\
\text { regressão no tratamento. }\end{array}$ \\
\hline $\begin{array}{l}17 \\
\text { SAVIETO(2016) }\end{array}$ & $\begin{array}{l}\text { Aplicação da teoria do cuidado } \\
\text { transpessoal de Jean Watson. }\end{array}$ & $\begin{array}{l}\text { Prover bons resultados no } \\
\text { tratamento do paciente por meio } \\
\text { da teoria do cuidado humano. }\end{array}$ \\
\hline $\begin{array}{l}18 \text { - SANTOS ET } \\
\text { AL. (2017) }\end{array}$ & $\begin{array}{l}\text { Os cuidados em enfermagem } \\
\text { analisados segundo a essência do } \\
\text { cuidado de Martin Heidegger. }\end{array}$ & $\begin{array}{l}\text { Mediante a teoria de Heidegger, } \\
\text { proporcionarmelhor cuidado ao } \\
\text { paciente. }\end{array}$ \\
\hline
\end{tabular}




\section{DISCUSSÃO}

A partir da leitura sucessiva dos artigos selecionados, a fim de realizar a presente revisão, e após o agrupamento das informações, pudemos comprovar como predominante nesses estudos a abordagem de quatro principais teorias: I. Teoria humanística; II. Teoria do autocuidado; III. Teoria do déficit do autocuidado; Teoria do cuidado humano.

\subsection{TEORIA HUMANÍSTICA}

Os cuidados paliativos na teoria humanística podem ser resumidos como tendo o foco naabordagem de diminuiçãodo sofrimento físico, psicossocial, espiritual e existencial, bem como estabelecer uma meta de comunicação abrangente e aberta com o paciente e seus familiares, promovendo assim uma melhora na qualidade de vida da pessoacom câncer de pulmão avançado 9 .

A teoria humanísticaconsiste numa forma de tratamento holístico e que visa preservar a integralidade física e moral do indivíduo, percebendo o paciente como um todo, colocando-se em seu lugar, suprindo assim suas necessidades ${ }^{6}$.

Por outro lado, o cuidado humanístico pode ser definido como:

Assim, como o conceito de humanização leva consigo a solidariedade e o respeito, o paciente fora de possibilidade de cura deve ter preservadas a sua autonomia e a sua dignidade. Vale ressaltar que quando o paciente percebe o cuidado humanizado pode exteriorizar suas vontades e sentimentos ao longo do processo de finitude, podendo alcançar a boa morte.

Outro aspecto importante a ser observado:

O cuidar em enfermagem exige que o profissional tenha um olhar abrangente e humanizado com o intuito de assistir à pessoa em sua integralidade, respeitando-a nos aspectos biopsicossociais e nas suas particularidades, deixando de valorizar somente a execução de técnicas e práticas específicas. ${ }^{5}$

Podemos resumir os cuidados paliativos humanísticos como sendo aqueles atos pautados na promoção da qualidade de vida e alívio da dor do paciente oncológico. Os cuidados paliativos humanístico são baseados na promoção da qualidade de vida do indivíduo e alívio da dor ${ }^{8}$.

\subsection{TEORIA DO AUTOCUIDADO}


O principal foco dessa teoria consiste em conceder meios para que o próprio paciente seja agente do cuidado, capaz de promover uma melhoria na sua qualidade de vida. Desse modo, o enfermeiro tem a função de usar uma linguagem simples para transmitir ao paciente os cuidados que aele possam ser delegados; cuidados simples, mas que possam preservar a atividade corporal e a melhoria daautoestima e qualidade de vida.Todas essas ações são fundamentais para o desenvolvimento apropriado do trabalho da equipe multiprofissional. A ausência desses fatores pode danificar a qualidade do atendimento.O enfermeiro, como líder da equipe, pode reduzir esse problema através do díalago sobre as angústias e medos, agregado ao acompanhamento psicológico $^{10}$.

Todavia o enfermeiro deve proporcionar uma maior aproximação do paciente oncológico com o tratamento, por intermédio de conversação, para que possa reconhecer suas dificuldades e necessidades, e dessa forma, promover uma melhoria na qualidade de vida, principalmente nesse momento de fragilidade ${ }^{10}$.

Contudo os cuidados paliativos confirmam a vida do indivíduo e encaram a morte como parte do seu cotidiano;não têm a função de adiar ou prolongar a morte, porém de aliviar seu sofrimento, proporcionando assim suporte a suas famílias no processo de encarar e enfrentar a morte de forma digna. Entretanto os cuidados paliativos têm o importante papel no quesito de saúde pública, principalmente quando se fala em cuidados paliativos em oncológicos, perante a epidemiologia destacada ${ }^{10}$.

É fundamental que os cuidados paliativos sejam agregados nas instituições de ensino, para que, dessa maneira,essas medidas paliativas sejam usadas de modo correto, tornando assim a formação profissional eficaz para atender melhor os familiares e o paciente, seja no hospital ou no domicílio ${ }^{10}$.

Os profissionais da enfermagem devem proporcionar uma orientação adequada ao paciente com câncer, para que assim os aspectos relacionados com a dor corporal sejam diminuídos. O paciente deve ser tratado com respeito e dignidade e deve ser considerado como um indivíduo pensante ${ }^{11}$.Mesmo que o câncer se encontre em estado terminal - o que sujeita o paciente a sentimentos como os de angústia, tristeza, impotência perante a morte -,cuidar da pessoa com câncer, apesar do sofrimento, poderá proporcionar um sentimento de gratidão ao profissional da saúde. Um simples ato de amor e compaixão poderá ajudar o paciente a sorrire a seguir em frente, e conceder melhor qualidade de vida no tempo que ainda lhe resta. É importante destacar a presença do profissional enfermeiro nas unidades de atendimento ao paciente com 
câncer, sendo indispensável sua formação e especialização, tendo em vista que seu planejamento e responsabilidade são fundamentais na coordenação da equipe ${ }^{11}$.

A enfermagem, bem como a equipe multidisciplinar,deve focar no paciente oncológico e seus familiares, que são parte da mesma situação. A equipe deve salientar o cuidado no paciente diagnosticado, porém deve também controlar sua atenção no ambiente familiar do paciente, para que possa estabelecer assistência integral e de qualidade $e^{12}$.

Para o paciente com neoplasia, o cuidar significa uma extensão emocional que aborda a terminalidade da vida e a confiança em Deus.O cuidado é um sentimento essencial ao ser humano, que percorre toda a humanidade, e que deve estar presente em nossas famílias e lares, no convívio com a sociedade, fortificando a relação entre cuidador e o ser cuidado. O cuidado é uma condição prévia de amor e saberes e de generosidade, o dirigir antecipado de todo o comportamento para que além de tudo o paciente seja livre e responsável, enfim, um ser humano digno ${ }^{11}$.

O processo de cuidar envolve uma relação de afeto, um processo interpessoal, resultando no sentido de confiança. Sendo assim, cuidar é servir, é perceber a essência do outro em pequenas atitudes, em pequenas palavras, em suas dificuldades, é um ato de amor por parte de quem o realiza. É necessário que o profissional tenha carinho com relação ao ser cuidado ${ }^{11}$.

È a prática de atividades iniciadas e executadas pelos indivíduos em seu própio beneficío para a manutenção da vida e do bem-estar [...].A capacidade de autocuidado só é afirmada quando o indíviduo é capaz de desempenhar a atividade de autocuidado para manter, restabelecer ou melhorar a sua saúde e bem-estar. $^{12}$

A teoria do autocuidado"tem como principal função diminuir os sintomas e as complicações das doenças e diminuir o tempo de recuperação e a taxa de hospitalização"

Podemos afirmar que o enfermeiro é essecial no autocuidado; é o resultado dos cuidados de enfermagem, contudo extremamente necessário para promoção da saúde e comodidade do individuo onde o enfermeiro tem destreza profissional e intercessão decisiva. ${ }^{13}$

O autocuidado é o conceito central da teoria do defícit do autocuidado. Para Orem, está prática contribui para o aprimoramento e amadurecimento dos indivíduos, que tem como objetivo a preservação da vida e o bem-estar pessoal ${ }^{14}$. 
No que respeita ao papel dos profissionais de saúde, nomeadamente dos enfermeiros, no sentido de participarem na gestão do autocuidado dos doentes, verificou-se a necessidade de alterar as estratégias standard de fornecimento de informação e ainda seguimento prévio aos tratamentos. ${ }^{15}$

\subsection{TEORIA DO DÉFICIT DO AUTOCUIDADO}

Consiste em explicar quando e porque a enfermagem se torna imprescindível ao indivíduo no processo de cuidar. Ela pode ser mais abrangente que a teoria anterior. Podemos definir o conceito de déficit de autocuidado como sendo a ligação entre o autocuidado e o quesito de cuidar, que está relacionado à teoria da enfermagem. Essa teoria pode representara principal função da enfermagem quando exercida em conexão com o sistema de enfermagem de uma maneira autêntica.

Sendo assim, a teoria do déficit do autocuidado é o núcleo central da teoria de Orem, sendo a teoria geral da enfermagem. Ela demonstra o quanto a enfermagem é necessária no cotidiano diário, principalmente quando o responsável por um paciente dependente encontra-se impossibilitado de exercer o cuidado. As principais funções da teoria do déficit do autocuidado são:

O termo déficit de autocuidado não significa uma "desordem humana", podendo no entanto estar relacionada "com a figura de uma desordem funcional ou estrutural do homem". O Déficit de Autocuidado é identificado a partir da relação entre as demandas de autocuidado e a incapacidade de ação dos indivíduos para atendê-las ${ }^{16}$.

\subsection{TEORIA DO CUIDADO HUMANO}

Segundo Watson, um dos instrumentos mais adequados para estabelecer e manter a importante relação de ajuda e confiança entre profissional e paciente é a empatia. A partir da verdadeira intenção de cuidar, é possível desenvolver uma relação empática, quando se reconhece o outro como quem vivencia sua experiência única de ser paciente e se expressa entendimento e aceitação através de linguagem verbal e não verbal ${ }^{17}$.

Na visão de Watson, a teoria do cuidado humano pode levar também em conta uma visão humanística do ser humano, independente dele ser profissional ou paciente, de modo que ambos possam ter seus princípios respeitados, para que assim fortaleça sua autonomia para que possam ser participante de um cuidado acolhedor ${ }^{17}$. 
Conforme Martin Heidegger, o refletir sobre o cuidado, talvez seja possível compreender que as atitudes dos profissionais de enfermagem precisam ser reavaliadas constantemente, pois, apesar de terem o cuidado como base da profissão, ainda se deparam com atitudes e práticas que os tornam mecanicistas. Precisam reconhecer o cuidado na perspectiva ontológica de sua existência, para que possam desenvolver uma prática de enfermagem humana e solidária, para além da técnica ${ }^{15}$. Assim, o cuidado em enfermagem, aproxima-se do modo ontológico de pensar do filósofo, pois o cuidar deve ultrapassar um simples acompanhamento do paciente, deve gerar construção de afinidade, na medida em que o enfermeiro e a pessoa cuidada vão se conhecendo. Nesse processo relacional, os dois compreendem e aprendem a estabelecer pontes que levam ao desvelamento do mundo de ambos, aprofundando dessa maneira, a relação de cuidado.

Por meio de diálogo há o favorecimento da escuta e um compartilhamento de significados, assim, o ser cuidado e o cuidador se fazem humano e torna-se possível construir uma relação compreensiva de cuidado. Podemos entender que na visão de Martin Heidegger a teoria do cuidado humano baseia-se em implicar as atividades desenvolvidas pelo profissional focando na habilidade, conhecimento, técnicae criatividade, atributos no qual contribuirá para manutenção, promoção e recuperação da dignidade e totalidade do indivíduo. Por fim, o cuidado na enfermagem tem base legal, competências, habilidades, técnicas, pensamento crítico, além de conhecimento e intuição. Entretanto, deve-se "estar consciente" e pensar sobre as formas de agir para que o cuidado em enfermagem alcance a perspectiva do cuidado apresentado por Heidegger. ${ }^{18}$ 


\section{CONSIDERAÇÕES FINAIS}

Os cuidados paliativos devem ser entendidos como um ato que vai além dos procedimentos técnicos. Existe a necessidade do envolvimento e compromisso com o outro, para que se possa harmonizar ações de humanização.A partir disso, os cuidados paliativos têm a função de ofertar ao paciente o cuidado antitumoral, que se destina à finalidade de melhorar sua qualidade de vidae monitorar o doente a uma doença que ameaça sua vida. Portanto esses cuidados devem ter a inspeção apropriada para prover a melhor compreensão do manejo de complicações e sinais estressantes do paciente, relacionados ao tratamento ou ao agravo da doença. Sendo assim, os cuidados de enfermagem têm o papel fundamental no tratamento do paciente oncológico;esses cuidados devem ser individualizados, visto que nesssa fase da vida as mudançasfisiológicas e psíquicas dos indivíduos com câncer o deixam debilitado e com uma perspectiva de vida menor. Devido a essa situação, há uma necessidade de aprimorar o relacionamento do paciente com sua equipe e familiares, abordando o processo de cuidar, focando principalmente na maneira como é dada a notícia, com a comprensibilidadeda forma de abordagem do assunto - abertura que é dada ao paciente e seus familiares para que possam dialogar sobre o sofrimento, dúvidas e a recuperação. Todavia espera-se que o resultado desta revisão possa contribuir para que os profissionais da enfermagem se apropriem da compreensão dos cuidados paliativos aqui abordados. Que possa também contribuir para seu aperfeiçoamento profissional em busca de uma melhora na qualidade de vida do paciente acometido de doenças avançadas como o câncer. 


\section{REFERÊNCIAS}

1. EVA, M. K. Palliativecareunits in lungcancer in the real-world setting: a single institution'sexperienceand its implications, AnnalsofPalativeMedicine,v. 6,n. 1, p. 613, 2017. Disponível em: <http://apm.amegroups.com/article/view/11516/1204 1>. Acesso em: 23 nov. 2018.

2. VINOD, K. Shaline, InternationalPatternsofRadiotherapyPractice for NonSmallCellLungCancer.Seminare in RadiationOncology, v. 25, n. 2, p. 143-150, abr. 2014. Disponível em: <https://doi.org/10.1016/j.semradonc.2014.11.001>. Acesso em: 23 nov. 2018.

3.MITCHE D. P.; KINNEDY, P. M. Bronchoscopy Management ofMalignantAirwayObstruction. Advances in Therapy, v. 31, n. 5, p. 512-538, 2004. Disponível em: <https://link.springer.com/article/10.1007\%2Fs12325-014-0122-z>. Acesso em: 24 nov. 2018.

4.SILVA,N. R. F. et al. Teorias de enfermagem aplicadas no cuidado a pacientes oncológicos:contribuição para a prática clínica do enfermeiro. Revista Uningá,Teresina, v. 55, n. 2, p. 59-71, jun. 2018. Disponível em: $<$ http://revista.uninga.br/index.php/uninga/article/view/1385>. Acesso em: 23 nov. 2018.

5. SILVA, M. M. et al.Cuidados paliativos na assistência de alta complexidade em oncologia:percepção do enfermeiro.Escola Ana Nery Revista de Enfermagem, Rio de Janeiro, v. 19, n. 3, p. 460-466, 2015. Disponível em: <http://www.redalyc.org/articulo.oa?id=127741627010>. Acesso em: 23 nov. 2018.

6. SANTOS, G. L.; MATOS, N. N. Cuidados paliativos aos pacientes oncológicos.Temas em Saúde, João Pessoa, v. 17,n. 1, 2017. Disponível em: <http://temasemsaude.com/wp-content/uploads/2017/05/17116.pdf >. Acesso em: 24 nov. 2018.

7. ROCHA, A. F. P. et al. O alívio da dor oncológica: estratégias contadas por adolescentes com câncer. Texto e Contexto Enfermagem, Florianópolis, v. 24, n. 1, jan./mar. 2015. Disponível em: <http://www.scielo.br/pdf/tce/v24n1/pt_0104-0707-tce24-01-00096.pdf>. Acesso em: 24 nov. 2018.

8. PEREIRA, C. S. et al. Significados dos cuidados paliativos para a qualidade da sobrevivência do paciente com câncer. Revista Brasileira de Cancerologia, Rio de Janeiro, v. 62, n. 3, p. 225-235, 2016. Disponível em: <http://www.inca.gov.br/rbc/ n_62/v03/pdf/05-artigo-significado-dos-cuidados-paliativos-para-a-qualidade-dasobrevivencia-do-paciente-oncologico.pdf>. Acessoem: 24 nov. 2018.

9. SHINDE, A. PalativeCare in lungcancer.CancerTreatmentandResearch, v. 170, 2018.Disponível em: 〈https://doi.org/10.1007/978-3-319-40389-2_11>. Acesso em: 24 nov. 2018. 
10. HERCOS, T. M. et al. O trabalho dos profissionais de enfermagem em unidade de terapia intensiva na assistência ao paciente oncológico. Revista Brasileira de Cancerologia, Rio de Janeiro, v. 60, n. 1, p. 51-58, 2014. Disponível em: <http:// www.inca.gov.br/rbc/n_60/v01/pdf/08-revisao-literatura-o-trabalho-dos-profissionaisde-enfermagem-em-unidades-de-terapia-intensiva-na-assistencia-ao-paciente oncologico.pdf>. Acesso em: 24 nov. 2018.

11. SCHIMIGUEL, J. O acolhimento do paciente oncológico: revisão bibliográfica. Saúde em Revista, Piracicaba, v. 15,n. 39, jan./mar. 2015. Disponível em: <https://www.metodista.br/revistas/revistas-unimep/index.php/sr/article/view/2375/14 61>. Acesso em: 24 nov. 2018.

12.NASCIMENTO, G. B. V.; ESPÍRITO SANTO, F. H.; NASCIMENTO, E. C. S. O familiar do cliente oncológico: uma revisão integrativa.Revista da Atenção Básica,v. 13,n. 45, p. 94-101, jul./set. 2015. Disponível em: <http://seer.uscs.edu.br/index.php/revista_ciencias_saude/article/view/3103>. Acesso em: 24 nov. 2018.

13. SANTOS, B.; RAMOS, A.; FONSECA, C. Da formação à prática: Importância das Teorias do Autocuidado no Processo de Enfermagempara a melhoria dos cuidados. JournalofAgingInovation, v. 6, n. 1, p. 51-54, 2017. Disponível em: <http://journa lofagingandinnovation.org/wp-content/uploads/6-Autocuidado-forma\%C3\% A7\%C3\% A3o.pdf>. Acesso em: 24 nov. 2018.

14. QUEIRÓS P.J. P. Et all;Autocuidado: o contributo teórico de Orem para a disciplina e profissão de enfermagem.Revista de Enfermagem Referência,v.4,n3 ,Dez 2014.Disponível emhttp://dx.doi.org/10.12707/RIV14081. Acesso em :24 de nov. 2018.

15. SILVA, J. M. C.; RIBEIRO, M. S. V. Estratégias de autocuidado das pessoas com doença oncológica submetidas a quimioterapia/radioterapia e a sua relação com $\mathrm{o}$ conforto. Revista Eletrônica de Enfermaria, n. 37, jan. 2015. Disponível em: <http:// scielo.isciii.es/pdf/eg/v14n37/pt_revision2.pdf>. Acesso em: 24 nov. 2018.

16. PEDROSA T. N.et all. Cuidado e autocuidado em oncologia significados para profissionais e usuários.Instituto Metodista de Ensino Superior n2, Jul-dez 2016.Disponível em file:///C:/Users/Barcellos/Desktop/6402-24319-2-PB.pdf.Acesso em 24 de nov.2018.

17. SAVIETO M.et al.Assistência em enfermagem e Jean Watson:Uma reflexão sobre empatia.Escola Anna Nery, v .20 n.1,Marc 2016. Disponível em:www.scielo.br/pdf/ean/v20n1/1414-8145-ean-20-01-0198.pdf. Acesso em 24 nov.2018. 
18. SANTOS, A. G. et al. O cuidado em enfermagem analisado segundo a essência do cuidado de Martin Heidegger. Revista Cubana de Enfermería, v. 33, n. 3,2017.Disponível em: 〈http://www.revenfermeria.sld.cu/index.php/enf/issue/view/27〉. Acesso em: 24 nov. 2018. 